\title{
Diseño participativo de un plan de social media en salud a través de metodología cualitativa. Evaluación de la experiencia del Hospital Universitario Reina Sofía
}

\section{Qualitative based social media plan participative design for health organizations. Evaluation of the Reina Sofia University Hospital experience}

\author{
Manuela López-Doblas; Olivia Pérez-Corral; David Gómez-Domínguez; Alina \\ Danet-Danet
}

Cómo citar este artículo:

López-Doblas, Manuela; Pérez-Corral, Olivia; Gómez-Domínguez, David; Danet-Danet, Alina (2019). “Diseño participativo de un plan de social media en salud a través de metodología cualitativa. Evaluación de la experiencia del Hospital Universitario Reina Sofía". El profesional de la información, v. 28, n. 2, e280224.

https://doi.org/10.3145/epi.2019.mar.24

Artículo recibido el 09-11-2018

Aceptación definitiva: 26-02-2019

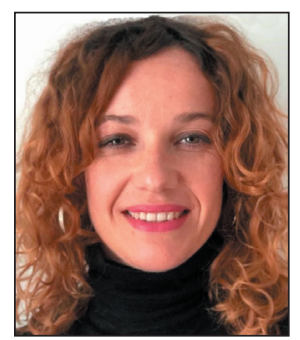

Manuela López-Doblas https://orcid.org/0000-0002-6741-625X

Escuela Andaluza de Salud Pública Cuesta del Observatorio, 4. 18011 Granada, España manuela.lopez.easp@juntadeandalucia.es

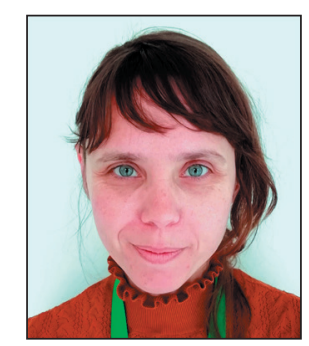

Olivia Pérez-Corral

https://orcid.org/0000-0003-4404-3662

Escuela Andaluza de Salud Pública Cuesta del Observatorio, 4. 18011 Granada, España olivia.perez.easp@juntadeandalucia.es

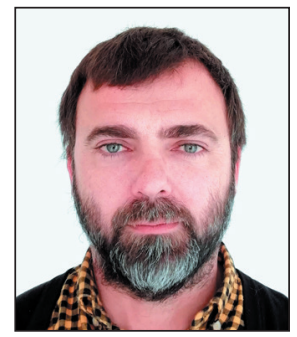

David Gómez-Domínguez https://orcid.org/0000-0003-2317-8189

Observatorio de la Infancia. Escuela Andaluza de Salud Pública Cuesta del Observatorio, 4. 18011 Granada, España david.gomez.easp@juntadeandalucia.es

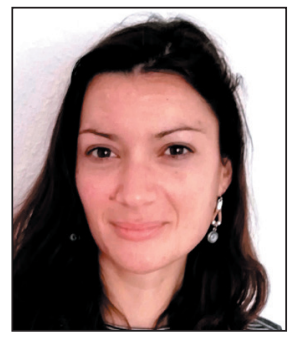

Alina Danet-Danet $\bowtie$ https://orcid.org/0000-0002-9025-982X

Ciber Epidemiología y Salud Pública (Ciberesp). Escuela Andaluza de Salud Pública

Cuesta del Observatorio, 4. 18011

Granada, España

alina.danet.easp@juntadeandalucia.es

\section{Resumen}

Se presenta una metodología cualitativa de diseño participativo para la realización del plan de social media (PSM) para organizaciones sanitarias, así como la evaluación de la experiencia en el Hospital Universitario Reina Sofía. La metodología de diseño colaborativo y multidisciplinar se desarrolló en cinco fases: identificación de agentes clave; consenso sobre objetivos, públicos, canales y líneas de contenido; análisis y devolución de resultados; diseño de (pre)campaña; elaboración colaborativa del PSM. La evaluación se realizó a través de la monitorización de presencia en redes (evaluación

Agradecimientos

Queremos agradecer al equipo de profesionales del Hospital Universitario Reina Sofía (HURS) que participaron en la elaboración del plan de social media y especialmente a Montemayor Mora Jiménez, Gema Timón Maldonado, José Manuel Rumbao Aguirre y Valle García Sánchez. 
cuantitativa de la comunicación externa) y análisis de contenido de entrevistas semiestructuradas al equipo directivo (evaluación cualitativa de la comunicación interna). Los resultados reflejan el impacto positivo en comunicación externa y la satisfacción con la metodología de co-diseño, evidenciando la importancia de la participación profesional y de la interdisciplinariedad, así como del apoyo directivo y de la profesionalización para la planificación de presencia en redes en el ámbito hospitalario.

\title{
Palabras clave
}

Redes sociales; Medios sociales; Plan de social media; PSM; Diseño participativo; Organizaciones sanitarias; Comunicación interna; Comunicación externa; Sistemas de comunicación en hospital; Comunicación en salud; Participación de los interesados; Hospitales.

\begin{abstract}
The participation-based design of a social media strategy for health organizations, and the evaluation of the experience in the Reina Sofía University Hospital are presented. The methodology of collaborative and multidisciplinary design was developed in five phases: identification of target actors; consensus on objectives, channels and content lines; results analysis and feedback; (pre)campaign design; collaborative elaboration of social media strategy. The evaluation included social media monitoring (quantitative evaluation of external communication) and content analysis of semi-structured interviews with hospital managers (qualitative evaluation of internal communication). Results show the positive impact on the external communication and the participants' satisfaction with the co-design, revealing that participatory and interdisciplinary approach, managerial support and professionalization represent the main aspects of social media presence for health organizations.
\end{abstract}

\section{Keywords}

Social media; Social media plan; SMP; Participation-based design; Health organizations; Internal communication; External communication; Hospital communication systems; Health communication; Stakeholders participation; Hospitals.

\section{Introducción}

En el ámbito de la salud se ha producido un incremento progresivo del uso de las redes sociales (Tonia, 2014; Ventola, 2014), justificado por su potencial de conectar e interactuar de manera inmediata con distintas audiencias, permitir la comunicación multidireccional y facilitar la creación de alianzas entre representantes de organizaciones sanitarias, ciudadanía y otras instituciones (Mangold; Faulds, 2009). En relación con las necesidades sociales de pacientes y usuarios, el uso de redes sociales aumenta las capacidades dinámicas de las organizaciones sanitarias, ya que transforma el acceso a la información y brinda más poder a pacientes y profesionales (Bjerglund-Andersen; Söderqvist, 2012; Andersen; Medaglia; Henriksen, 2012). Además facilita el diálogo público, la participación y colaboración, así como la monitorización de la calidad y eficiencia (Chetley, 2006; Vance; Howe; Dellavalle, 2009).

El uso de redes sociales aumenta las capacidades dinámicas de las organizaciones sanitarias, ya que transforma el acceso a la información y brinda más poder a pacientes y profesionales

Usadas de manera eficaz, estas tecnologías tienen utilidad para la vigilancia y educación para la salud (Kass-Hout; Alhinnawi 2013), en casos de emergencia o alerta sanitaria, con fines de investigación y transferencia del conocimiento (Mairs et al., 2013), en la intervención para la salud y en procesos de cambio o adopción de hábitos de vida saludables (Vance; Howe; Dellavale, 2009; Merchant; Elmer; Lurie, 2011), así como para la creación de redes (Roman, 2014; Bjerglund-Andersen; Söderqvist, 2012).

Para la comunidad sanitaria, las redes sociales son un medio para mejorar la calidad y práctica profesional (Ventola, 2014; Bernhardt; Alber; Gold, 2014) a bajo costo (Gomes; Coustasse, 2015; Blázquez-Fernández; Cantarero-Prieto; Pascual-Sáez, 2015). En la comunicación con pacientes y usuarios permiten la interacción en tiempo real y representan herramientas y espacios de acceso e intercambio de información, de participación y expresión (Anthenius; Tates; Nieboer, 2013; Krowchuk; Lane; Twaddell, 2010).

Entre profesionales de la salud se usan para difundir resultados, discusiones y trabajo en red, para enseñanza e investigación (Bjerglund-Andersen; Söderqvist, 2012; Chretien; Kind 2013) y para la interacción biomédica (Grindrod et al., 2014; Grajales et al., 2014; Von-Muhlen; Ohno-Machado, 2012).

\author{
Entre profesionales de la salud, las redes \\ sociales se usan para difundir resulta- \\ dos, discusiones y trabajo en red, para \\ enseñanza e investigación
}

Los obstáculos y limitaciones en el uso de las redes sociales por parte de las organizaciones sanitarias se asocian principalmente a cuestiones legales y de privacidad (Bjerglund-Andersen; Söderqvist, 2012; Andersen; Medaglia; Henriksen, 2012, Chretien; Kind, 2013) y a riesgos potenciales para pacientes y profesionales, que incluyen: 
- distribución de información de mala calidad;

- daños a la imagen profesional;

- violación de la privacidad de pacientes y de los límites personales y profesionales;

- licencias; o

- problemas legales (Ventola, 2014).

Desde la propia comunidad médico-científica se han observado pautas de resistencia al uso de las redes sociales, fundamentadas por la sensación de falta de control, dificultad para evaluar los beneficios, desconfianza respecto a la veracidad y fiabilidad de la información (Bjerglund-Andersen; Söderqvist, 2012; Research Information Network, 2010; Castilla, 2016), o la preocupación por posibles efectos negativos, como falsas acusaciones, críticas dañinas (Bjerglund-Andersen; Söderqvist, 2012), autoría ciega, falta de cita en la fuente y presentación de la opinión como un hecho (Vance; Howe; Dellavalle, 2009).

Ante esta serie de desventajas, las instituciones sanitarias y las organizaciones profesionales internacionales han emitido pautas de prevención y guías de buenas prácticas (Ventola, 2014) orientadas a una mejor gestión del riesgo a nivel organizacional, clínico y legal (Lambert; Barry; Stokes, 2012; Househ, 2013), y a la protección de los derechos de pacientes y profesionales (Dizon et al., 2012; Childs; Martin, 2012). En España existen recomendaciones generales para el uso de redes sociales, así como guías de uso y estilo elaboradas en varias comunidades autónomas (Juárez-Giménez; Fernández-Lisón; Monte-Boquet, 2014).

En Andalucía, las directrices del IV Plan andaluz de salud (2013-2020) hacen hincapié en la transparencia en la gestión y comunicación corporativa, la mejor atención sanitaria y difusión del conocimiento y la necesidad de recoger y atender las demandas ciudadanas (Rando-Cueto; De-las-Heras-Pedrosa, 2016). Dentro de este marco jurídico e institucional, las redes sociales son un instrumento de máxima utilidad, confirmada por la puesta en marcha de RedSaludAndalucía, una plataforma impulsora de las redes sociales públicas sanitarias (Rando-Cueto; De-las-Heras-Pedrosa, 2016).

A nivel de organizaciones hospitalarias, los estudios disponibles no son concluyentes con respecto al uso de redes sociales, si bien reflejan un infra-uso en Europa y Estados Unidos (Van-de-Belt et al., 2012; Moorhead et al., 2013; Vanzetta et al., 2014; Griffis et al., 2014, Richter; Muhlestein; Wilks, 2014), a la vez que destacan su escasa eficiencia a nivel internacional (Yang et al., 2018; Zhang et al., 2018). Sin embargo, también se conocen hospitales con gran visibilidad y reconocimiento de marca en los medios sociales: Mayo Clinic, Cleveland Clinic o Mount Sinai Hospital, todos de EUA (Medina-Aguerrebere, 2018; Medina-Aguerrebere; Buil; Heath, 2016).

En España sólo la mitad de los hospitales utilizan Facebook, Twitter o YouTube como canales de comunicación (Costa-Sánchez; Túñez-López; Videla-Rodríguez, 2016). La mayor presencia en redes se registra en hospitales públicos y de mayor tamaño (Bermúdez-Tamayo et al., 2013) y la calidad de los sitios web es baja (Calvo-Calvo, 2014). Pese a una apuesta generalizada por las redes sociales, los hospitales siguen una pauta de infra-comunicación de la identidad corporativa, de poca variedad de redes sociales utilizadas, así como de escasos contenidos y aplicaciones difundidas, en el contexto de una limitada profesionalización de la presencia corporativa en medios sociales (Medina-Aguerrebere, 2018).

Con respecto a los hospitales andaluces, Rando-Cueto (2014) observó una falta de coherencia entre la intencionalidad de la información emitida en redes y sus resultados y consecuencias, así como una escasa participación de los hospitales andaluces en redes sociales (Rando-Cueto; De-las-Heras-Pedrosa, 2016), con eco en la falta de diálogo y la baja participación ciudadana en las cuentas de Twitter de instituciones hospitalarias, pese a sus demandas e interés (Rando-Cueto; Paniagua-Rojano; De-las-Heras-Pedrosa, 2016).

En el sector hospitalario, donde la interacción entre profesionales y pacientes es continua y determinante para satisfacer las necesidades de información y asistencia sanitaria (Medina-Aguerrebere, 2018), la responsabilidad sobre la comunicación institucional concierne a toda la organización, requiere la participación y compromiso, y a la vez tiene impacto, tanto sobre los clientes internos (el personal sanitario) como externos (pacientes, ciudadanos y otros públicos) (Illia; Balmer, 2012).

Los principios del diseño participativo, base de los proyectos de co-creación de reciente aplicación en la comunicación en organizaciones sanitarias, desvelan las ventajas de la colaboración de las personas participantes a la largo de todas las fases de diseño, implementación y evaluación de un proyecto (Langley; Wolstenholme; Cooke, 2018; Smith; Bossen; Kanstrup, 2017; Greenhalgh et al., 2016; Rycroft-Malone et al., 2016).

Las organizaciones hospitalarias deben priorizar la dimensión relacional más allá de la tecnológica en su comunicación. Trasladado al marco teórico, el concepto de Groundswell, desarrollado por Li y Bernoff (2008), propone integrar el uso de redes sociales dentro de la estrategia comunicacional de la organización, tener presente no sólo su propósito y objetivos, sino también los perfiles de las audiencias a quienes se dirigen y la elección de los social media más apropiados (Chou et al., 2009).
Las organizaciones hospitalarias deben priorizar la dimensión relacional más allá de la tecnológica en su comunicación 
Dentro de este marco que prioriza la comunicación institucional como responsabilidad de un equipo multidisciplinar y basado en la implicación de todo el personal hospitalario, el objetivo de este trabajo es diseñar y evaluar un plan de social media (en adelante, PSM) para una organización hospitalaria, partiendo de una metodología cualitativa de diseño participativo y multidisciplinar.

\section{Metodología}

A continuación se contextualiza y describe la metodología llevada a cabo:

\subsection{Diseño}

Proceso participativo de diseño colaborativo y triangulación de métodos y técnicas para la creación y puesta en marcha de un PSM, basado en la implicación de todas las personas participantes y la búsqueda de consenso en todas las fases del proceso.

\subsection{Organización hospitalaria}

EI PSM se realizó en el Hospital Universitario Reina Sofía de Córdoba (HURS), complejo hospitalario perteneciente al Servicio Andaluz de Salud, en el que trabajan 4.970 profesionales, organizados en 41 unidades de gestión clínica, que atienden a una población de referencia de 455.000 personas (HURS, 2016).

\subsection{Participantes}

En el diseño del PSM participaron de manera triangular representantes de la dirección y Unidad de Comunicación de la organización hospitalaria (3 participantes), profesionales de plantilla del hospital (16 participantes) y un equipo de personas expertas en metodologías y comunicación sanitaria (equipo asesor: 3 personas).

\subsection{Antecedentes}

Un trabajo previo elaborado por la responsable de comunicación del centro y realizado en el marco de una actividad formativa coordinada por miembros del equipo asesor constituyó el punto de partida y apuesta por compartir y poner el producto al servicio de la organización, con utilidad en cuanto al acercamiento teórico y fase previa de descripción y diagnóstico.

\subsection{Fases metodológicas}

Para dar respuesta al objetivo de diseñar el PSM a través de una metodología cualitativa con diseño participativo, se llevaron a cabo 5 fases, durante los meses de enero-febrero de 2018:

\section{Fase 1}

- Objetivo: identificación de los agentes claves en el proceso.

- Participantes: equipo de dirección: gerente del HURS, director médico y responsable de la Unidad de Comunicación del centro.

- Técnica: entrevistas semiestructuradas.

La selección de 16 profesionales sanitarios participantes se realizó en colaboración con la dirección del centro, a partir de perfiles definidos en base a los siguientes criterios de segmentación: sexo, edad, categoría profesional, área de trabajo, manejo de TICs y uso de redes sociales (tabla 1)

\section{Fase 2}

Tabla 1. Perfiles de profesionales participantes
\begin{tabular}{|l|l|l|c|}
\hline \multicolumn{1}{|c|}{ Ámbito/unidad } & Perfil/ categoría profesional & \multicolumn{1}{|c|}{ Sexo } & N. \\
\hline Informática & Profesional & Hombre & 5 \\
\hline Administración & Profesional & Mujer & 1 \\
\hline \multirow{4}{*}{ Comunicación } & Profesional & Mujer & 2 \\
\cline { 2 - 4 } & Profesional & Hombre & 1 \\
\cline { 2 - 4 } & Responsable & Mujer & 1 \\
\hline \multirow{4}{*}{ Dirección/ Gerencia } & Responsable (medicina) & Mujer & 1 \\
\cline { 2 - 4 } & Responsable (medicina) & Hombre & 2 \\
\cline { 2 - 4 } & Responsable (enfermería) & Hombre & 1 \\
\hline \multirow{3}{*}{ Asistencial } & Profesional (medicina) & Hombre & 3 \\
\cline { 2 - 4 } & Profesional (medicina) & Mujer & 1 \\
\hline Formación & Profesional & Hombre & 1 \\
\hline Actividades motivacionales & Profesional & Mujer & 1 \\
\hline
\end{tabular}

- Objetivo: validación y ampliación consensuada de objetivos, públicos diana, canales y principales líneas de contenido vinculadas a los principales valores de la organización.

- Participantes: equipo de dirección y profesionales sanitarios (19 participantes).

- Técnica: grupo de trabajo, conferencia de consenso, lluvia de ideas y técnica DAFO (debilidades, amenazas, fortalezas, oportunidades).

Esta fase se llevó a cabo en los siguientes pasos:

1. Análisis de situación de partida.

2. Identificación consensuada de objetivos, públicos y canales. 
3. Identificación de necesidades emergentes: recopilar y ordenar los recursos y contenidos existentes, identificar profesionales activos en redes, disponer de un protocolo de actuación en caso de crisis y una guía de estilo del HURS en redes.

4. Identificación de las principales líneas de contenido para el diseño de futuras acciones 2.0.

5. identificación de perfiles no oficiales vinculados al HURS.

\section{Fase 3}

- Objetivo: presentación de los principales resultados de la fase 2.

- Participantes: equipo de dirección.

- Técnica: sesión informativa y grupo de trabajo.

\section{Fase 4}

- Objetivo: diseño colaborativo de la precampaña y la campaña de lanzamiento en redes sociales (\#soyreinasofía).

- Participantes: equipo de dirección y profesionales sanitarios (19 participantes).

- Técnica: grupo de trabajo, conferencia de consenso, lluvia de ideas, dinámica de divergencia y convergencia.

Se dividió al grupo en 3 subgrupos con la misión de que cada uno de ellos diseñase una campaña con eslogan (\#hashtag), plazos y acciones, de forma argumentada. Tras la exposición de las tres propuestas (dinámica de divergencia), se definió la campaña definitiva que englobó acciones de las tres propuestas presentadas (dinámica de convergencia).

Se identificaron posibles aliados locales (instituciones y personas conocidas) y del entorno sanitario 2.0, potenciales colaboradores para la pre-campaña y campaña.

\section{Fase 5}

- Objetivo: elaboración del PSM en base a los resultados del grupo.

- Participantes: responsable de la Unidad de Comunicación y equipo asesor.

- Técnica: sesión informativa y grupo de trabajo.

Partiendo de los objetivos, públicos, canales y líneas de contenido definidos, se trabajaron las acciones puntuales y periódicas, protocolo de crisis, guía de estilo y el plan de evaluación y los materiales que se consideraron necesarios para el PSM.

\subsection{Evaluación}

La evaluación del impacto del PSM se ha abordado a partir de un diseño metodológico mixto y se llevó a cabo en dos momentos clave:

- durante las semanas de lanzamiento de la precampaña y la campaña: evaluación de la comunicación externa a través de monitorización cuantitativa;

- en un momento posterior, una vez trascurridos 8 meses de la campaña: evaluación de la comunicación interna a través de técnicas cualitativas.

El impacto de la comunicación externa se realizó a través de la monitorización del hashtag \#soyreinasofía en Facebook y YouTube (a través de las herramientas disponibles en la plataforma) y Twitter (con KeyHole y TweetBinder), así como a través del seguimiento de la repercusión en medios periodísticos digitales.

Para medir el impacto en la comunicación interna, así como para conocer la experiencia vivida y contada por las propias personas participantes, se usó metodología cualitativa. Se realizaron dos entrevistas semiestructuradas al equipo de dirección del HURS durante el mes de octubre 2018, contando con una persona con experiencia en la metodología cualitativa y externa al proceso de diseño. Las entrevistas, con una duración media de una hora se grabaron en formato audio y posteriormente se analizó su contenido con el software N.Vivo. El análisis se realizó por parte de la persona externa, incluyó precodificación, codificación, definición de categorías y triangulación entre los miembros del equipo asesor.

\section{Resultados}

\subsection{Modelo de metodología cualitativa basada en diseño participativo}

El diseño del PSM para el HURS se configuró en un modelo con las siguientes fases (tabla 2):

- creación de equipo de trabajo y cronograma;

- elección de nombre e imagen corporativa;

- análisis de situación;

- definición de objetivos;

- identificación de públicos;

- selección de canales 2.0;

- priorización de líneas de contenido/valores;

- diseño de campaña de lanzamiento;

- devolución de resultados. 
Tabla 2. Fases del modelo y resultados del diseño del PSM

\begin{tabular}{|c|c|c|}
\hline Fase & Técnica participativa & Resultados (@HUReinaSofía) \\
\hline $\begin{array}{l}\text { Creación de equipo de } \\
\text { trabajo y cronograma }\end{array}$ & $\begin{array}{l}\text { Entrevistas con responsables de la } \\
\text { Unidad de Comunicación y el equipo de } \\
\text { dirección }\end{array}$ & $\begin{array}{l}\text { Grupo multidisciplinar de } 19 \text { profesionales. } \\
\text { Cronograma. }\end{array}$ \\
\hline $\begin{array}{l}\text { Nombre e imagen } \\
\text { corporativa }\end{array}$ & $\begin{array}{l}\text { Grupo de trabajo multidisciplinar: } \\
\text { técnicas participativas/de consenso } \\
\text { (Apoyo de profesionales expertos en } \\
\text { diseño gráfico) }\end{array}$ & @HUReinaSofía en todos los canales. \\
\hline Análisis de situación & $\begin{array}{l}\text { Grupo de trabajo multidisciplinar: } \\
\text { Técnicas participativas/de consenso, } \\
\text { DAFO y recogida de información a } \\
\text { través de buscadores de redes sociales } \\
\text { generalistas (Facebook, Twitter, You- } \\
\text { Tube) }\end{array}$ & $\begin{array}{l}\text { Análisis de situación: } \\
\text { YouTube: } 2 \text { canales (sólo } 1 \text { oficial y otro centrado en trasplantes y donación } \\
\text { de órganos). } \\
\text { Facebook: } 8 \text { perfiles no oficiales. } \\
\text { Twitter: } 4 \text { perfiles no oficiales. } \\
\text { DAFO: } \\
\text { Debilidades: formación, disponibilidad profesional, privacidad del paciente, } \\
\text { resistencias internas, selección de contenidos. } \\
\text { Amenazas: Mal uso de las RRSS, alta competencia, presiones externas. } \\
\text { Fortalezas: formación de profesionales, motivación de los profesionales, } \\
\text { marca HURS, contenidos de interés. } \\
\text { Oportunidades: Servicios y ventajas de estar en RRSS, existencias de alia- } \\
\text { dos, y experiencias previas de las que aprender. }\end{array}$ \\
\hline $\begin{array}{l}\text { Definición de } \\
\text { objetivos }\end{array}$ & $\begin{array}{l}\text { Grupo de trabajo multidisciplinar: } \\
\text { técnicas participativas/de consenso }\end{array}$ & $\begin{array}{l}\text { Objetivos cuantitativos: visibilidad, visitas web, interactividad, inmediatez, } \\
\text { alianzas. } \\
\text { Objetivos cualitativos: liderazgo e imagen, transparencia, reconocimiento, } \\
\text { educación para la salud, escucha, accesibilidad, humanización. }\end{array}$ \\
\hline $\begin{array}{l}\text { Identificación de públi- } \\
\text { cos objetivos }\end{array}$ & $\begin{array}{l}\text { Grupo de trabajo multidisciplinar: } \\
\text { técnicas participativas/de consenso }\end{array}$ & $\begin{array}{l}\text { Públicos internos: } \\
\text { Se identificaron } 9 \text { públicos diferentes, de los que se priorizaron: profesiona- } \\
\text { les sanitarios y no sanitarios, empresas proveedoras, Instituto Maimónides } \\
\text { de Investigación Biomédica de Córdoba (Imibic), otros hospitales provinciales } \\
\text { Consejería de Salud/Servicio Andaluz de Salud. } \\
\text { Públicos externos: } \\
\text { Se identificaron } 15 \text { tipos de públicos, entre los que se priorizaron: ciuda- } \\
\text { danía en general, pacientes, familiares y personas cuidadoras, medios de } \\
\text { comunicación, otras instituciones sanitarias. }\end{array}$ \\
\hline $\begin{array}{l}\text { Selección de } \\
\text { canales } 2.0\end{array}$ & $\begin{array}{l}\text { Grupo de trabajo multidisciplinar: } \\
\text { técnicas participativas/de consenso y } \\
\text { exposición características específicas } \\
\text { de redes }\end{array}$ & $\begin{array}{l}\text { Página en Facebook. } \\
\text { Perfil en Twitter. } \\
\text { Canal en YouTube. } \\
\text { En fases futuras se contempla la posibilidad de incorporar nuevas redes. }\end{array}$ \\
\hline $\begin{array}{l}\text { Priorización de líneas } \\
\text { de contenido/valores }\end{array}$ & $\begin{array}{l}\text { Grupo de trabajo multidisciplinar: } \\
\text { técnicas participativas/de consenso }\end{array}$ & $\begin{array}{l}\text { Liderazgo e imagen. } \\
\text { Transparencia. } \\
\text { Reconocimientos a profesionales HURS. } \\
\text { Escucha. } \\
\text { Humanización. }\end{array}$ \\
\hline $\begin{array}{l}\text { Diseño campaña de } \\
\text { lanzamiento }\end{array}$ & $\begin{array}{l}\text { Grupo de trabajo multidisciplinar: } \\
\text { técnicas participativas/de consenso y } \\
\text { dinámica de divergencia y convergen- } \\
\text { cia a través de } 3 \text { subgrupos de trabajo. }\end{array}$ & $\begin{array}{l}\text { Precampaña y campaña \#SoyReinaSofía: } \\
2 \text { semanas de duración. } \\
\text { Acciones on line y presenciales: photocall, vídeos de profesionales, famosos } \\
\text { y aliados } 2.0 \text {, etc. vídeo \#SoyReinaSofía y rueda de prensa. }\end{array}$ \\
\hline $\begin{array}{l}\text { Devolución de resulta- } \\
\text { dos a la organización }\end{array}$ & $\begin{array}{l}\text { Trabajo del equipo asesor: análisis de } \\
\text { resultados y redacción de informes } \\
\text { definitivos }\end{array}$ & $\begin{array}{l}\text { Informe final: plan social media del @HUReinaSofía. } \\
\text { Informe de impacto de la campaña de lanzamiento en redes sociales. }\end{array}$ \\
\hline
\end{tabular}

\subsection{Campaña de lanzamiento en redes sociales}

La metodología empleada en el HURS para el diseño participativo de lanzamiento en redes tuvo como resultado la iniciativa \#SoyReinaSofía que, partiendo del componente lúdico y humorístico de los diferentes significados del término "Reina Sofía", se llevó a cabo con acciones online y presenciales para incrementar la visibilidad de la salida a redes sociales de la organización sanitaria.

Con este eslogan se pretendía reforzar la marca del hospital y la identificación y vinculación de profesionales y ciudadanos con el centro. La campaña de lanzamiento duró dos semanas y se estructuró en dos intervalos.

La primera semana se centró en la precampaña, antes de la salida en redes, y su principal objetivo fue generar expectación y ruido en redes sociales con el hashtag \#SoyReinaSofía. La acción principal de esta primera parte de la campaña fue el diseño y creación de un photocall que representaba los diferentes significados de 'Reina Sofía': hospital, museo, y la Reina Sofía. Este photocall se ubicó durante una semana en espacios emblemáticos de la ciudad, invitando a compartir las fotos realizadas en las redes sociales con el hashtag \#SoyReinaSofía. 
El segundo período de la campaña arrancó el 1 de marzo de 2018, con el lanzamiento en Facebook, Twittery YouTube del Hospital Reina Sofía (@HUReinaSofía). La acción central fue la realización de un vídeo con participación de profesionales del hospital y ciudadanos, que recogía los principales valores del centro.

https://youtu.be/6imXa5Hby7A

Como otra acción de continuidad, se invitó a personajes famosos, otros profesionales del hospital, referentes en salud 2.0 y ciudadanos en general, a enviar su propio vídeo con el eslogan "\#SoyReinaSofía desde... (una fecha concreta vinculada a su relación con el hospital) y “A partir de ahora seré Reina Sofía cada día en redes sociales" (tabla 3).

Tabla 3. Objetivos y acciones de la campaña

\begin{tabular}{|c|c|}
\hline \multicolumn{2}{|l|}{ Campaña \#SoyReinaSofía. 22/02/2018 - 07/03/2018 } \\
\hline \multicolumn{2}{|c|}{$\begin{array}{l}\text { Objetivo general: Dar a conocer la presencia del Hospital Universitario Reina Sofía en redes sociales, concretamente en Facebook, Twitter y } \\
\text { YouTube. }\end{array}$} \\
\hline \multicolumn{2}{|l|}{$\begin{array}{l}\text { Precampaña: } \\
\text { (Antes de salir a redes) } \\
22 / 02 / 2018-28 / 02 / 2018\end{array}$} \\
\hline Objetivos específicos & Acciones \\
\hline $\begin{array}{l}\text { - Generar expectación en torno al hashtag \#SoyReinaSofía. } \\
\text { - Lograr presencia de \#SoyReinaSofía en redes. }\end{array}$ & $\begin{array}{l}\text { Acción 1: Photocall \#SoyReinaSofía en espacios clave. } \\
\text { Acción 2: Publicación de noticias sobre el photocall. }\end{array}$ \\
\hline \multicolumn{2}{|l|}{$\begin{array}{l}\text { Campaña } \\
\text { (Desde el lanzamiento a redes) } \\
01 / 03 / 2018-07 / 03 / 2018\end{array}$} \\
\hline Objetivos específicos & Acciones \\
\hline $\begin{array}{l}\text { Cualitativos: } \\
\text { - Presentar PSM HURS en Facebook, Twitter y YouTube. } \\
\text { - Reforzar los valores de la marca HURS. } \\
\text { - Reconocer la labor de los profesionales del HURS. } \\
\text { - Dar a conocer vivencias de pacientes, familiares, etc. en el HURS. } \\
\text { Cuantitativos: } \\
\text { - Incrementar la participación en la campaña. } \\
\text { - Lograr un mínimo de } 150 \text { seguidores y/o suscriptores en cada red } \\
\text { social una vez finalizada la campaña. } \\
\text { - Lograr impacto en medios de comunicación, al menos } 2 \text { noticias en } \\
\text { medios locales, y } 1 \text { en medios autonómicos. }\end{array}$ & $\begin{array}{l}\text { Acción 3: Elaboración del vídeo de la campaña \#SoyReinaSofía, con } \\
\text { participación de profesionales y ciudadanía. } \\
\text { https://youtu.be/6imXa5Hby7A } \\
\text { Acción 4: Rueda de prensa sobre la presentación del PSM y la cam- } \\
\text { paña \#SoyReinaSofía. } \\
\text { Acción 5: Vídeos breves \#SoyReinaSofía, con personas famosas, } \\
\text { profesionales, ciudadanos, etc. }\end{array}$ \\
\hline
\end{tabular}

\subsection{Evaluación de la campaña}

La monitorización cuantitativa se realizó para evaluar la comunicación externa de la campaña de lanzamiento.

Durante la precampaña se lanzaron 127 tweets procedentes de 40 usuarios, con un alcance de 31.859 usuarios únicos y un total de 58.579 impresiones. Además tuvo dos impactos en medios de comunicación, uno en prensa digital (El día de Córdoba) y otro en radio (Radio Córdoba - Cadena SER).

En el período de campaña se publicaron 179 tweets con el hashtag \#SoyReinaSofía (160 autores usuarios únicos), que fueron vistos en más de 720 mil ocasiones (impresiones). Durante el período de campaña se consiguieron 864 seguidores, con una distribución igualada por sexo (hombres un $56 \%$ y mujeres un $44 \%$ ) y con procedencia mayoritaria de Andalucía (el 60\%, de los cuales el $38 \%$ fueron de la provincia de Córdoba).

En Facebook durante el período de campaña se consiguieron 636 seguidores, la página fue visitada en 2.557 ocasiones por 81.586 personas (alcance) y las publicaciones tuvieron un total de 22.267 interacciones. Los seguidores fueron un $81 \%$ mujeres, con intervalo de edad de 25 a 54 años localizadas en Córdoba y provincia.

En YouTube se consiguieron 157 suscriptores, se compartieron 61 vídeos con el hashtag \#SoyReinaSofía, configurando la lista de reproducción denominada "Campaña \#SoyReinaSofía", que contó con más de 2 mil visualizaciones.

La campaña también tuvo impacto en medios como ABC Córdoba, El día de Córdoba, Ahora Córdoba y Europa Press.

El vídeo de la campaña fue distribuido directamente a través de Twitter y Facebook, además de ser colgado en el canal de YouTube del HURS donde alcanzó más de 1.000 visualizaciones. Las reproducciones según el canal mostraron el mayor impacto en Facebook (compartido 600 veces, más de 22 mil reproducciones, 1.459 "Me gusta" -hola 257 en publicación y 1.202 en contenido compartido- y 178 "Me encanta" -46 en publicación y 132 en contendido compartido-). En Twitter el vídeo registró 4.623 reproducciones, 117 "Me gusta” (MG) y 77 veces fue retweeteado.

La evaluación del impacto a los ocho meses del lanzamiento de la campaña (noviembre 2018) reveló 2.631 seguidores en Twitter, 24.410 en Facebook (con 24.534 “Me gusta”) y en YouTube 314 suscriptores, 292 vídeos y 43.581 visualizaciones. 
La evaluación cualitativa se centró en el impacto del PSM en la comunicación interna. Los resultados del análisis de contenidos de las entrevistas reflejaron un alto grado de satisfacción con la creación y alcance del PSM. Los participantes resaltaron que la salida a redes era una necesidad imperiosa en el HURS, para la cual fue determinante el apoyo directivo y la dotación económica.

Según los entrevistados, la consideración del PSM como "éxito" partió de las ventajas de la metodología de codiseño participativo y tuvo como principales resultados el impacto positivo en la comunicación interna y en la relación con los ciudadanos y otros agentes sociales.

El acierto en el uso de metodología de codiseño participativo se argumentó en base a la interdisciplinariedad y heterogeneidad de perfiles profesionales participantes, que aseguraron la óptima identificación de objetivos, públicos, canales y líneas de contenido, a la vez que permitieron rentabilizar el talento, la pluralidad, generar sentido de pertenencia y favorecer alianzas. Se consideró una clave de éxito haber dado continuidad a las sesiones presenciales a través de la creación de un grupo activo de Whatsapp, liderado por la Unidad de Comunicación.

"El haber convertido a profesionales que podían haber sido enemigos en aliados. Eso es fundamental porque hay profesionales que en el momento de salir a redes sabían más de esto que el equipo de comunicación. Haberlos integrado es clave para que sintieran que este trabajo que ellos habían hecho antes se proyectaba y que su talento servía para enriquecernos a todos" (profesional de comunicación).

El impacto de la presencia en redes en la comunicación interna se describió como un "antes y un después", destacando la mayor accesibilidad del personal sanitario a toda la información institucional, el aumento de la capacidad de comunicación y visibilidad de las Unidades de Gestión Clínica (UGC) y de su colaboración con la Unidad de Comunicación. Por parte de esta Unidad, se asumió de manera explícita y responsable la planificación y liderazgo de la estrategia de comunicación, basada en un enfoque dinámico y participativo, garantizado a través de reuniones con profesionales, orientadas a identificar conjuntamente contenidos a difundir de manera estratégica en redes, lo que simbólicamente se describió como la relación entre "corresponsales" y "redacción central" (profesional de comunicación).

"Antes, la Unidad de Comunicación iba detrás de las UGC buscando información para tener noticias, pero ahora que las Unidades ya ven cómo pueden tener presencia, se ha generado un efecto llamada y las Unidades de manera proactiva acuden a la Unidad de Comunicación porque quieren tener visibilidad" (profesional de dirección).

"Los profesionales lo han visto como una oportunidad. Ahora todos tienen oportunidad de salir en Red y no sólo las unidades que tradicionalmente tienen más presencia como cardiología o trasplantes" (profesional de dirección).

En relación con la comunicación externa se señaló el valor de las redes para favorecer el acercamiento entre hospital y ciudadanos, facilitando el acceso a sus expectativas y necesidades. Así mismo se refirió una mejor visibilidad del centro y la creación de sinergias y alianzas con otras personas y entidades públicas, con impacto en el aumento del prestigio y la reputación del Hospital.

Por último, las claves para la implantación de un diseño participativo de un PSM fueron, en el caso del HURS:

- presencia activa y el liderazgo ejercido desde la Unidad de Comunicación;

- apoyo constante de la Dirección;

- asesoramiento externo y especializado;

- planificación colaborativa de la estrategia;

- alianzas con profesionales presentes en redes como facilitadores para la acogida durante la campaña y con el personal sanitario del centro como generadores de contenidos;

- creación de un equipo y protocolo de gestión de crisis;

- continuidad y seguimiento por parte del grupo propulsor del PSM.

\section{Discusión}

La descripción de la metodología y evaluación del plan de social media del HURS pone de manifiesto la utilidad y ventajas del codiseño participativo de la presencia en redes de una organización sanitaria, con impacto positivo en la comunicación interna y externa, así como en la satisfacción y vivencia del proceso por parte de las personas participantes.

Con respecto al modelo de diseño colaborativo, cabe comentar que la participación de profesionales de la propia organización sanitaria garantizó una definición de objetivos, públicos y líneas de contenidos adecuados a la realidad institucional, basada en los conocimientos y valores de la misma. La incorporación de profesionales hospitalarios a la toma de decisión sobre la estrategia representó un primer mecanismo de integración y fomentó el reparto de visibilidad y 
contribución de diferentes unidades y perfiles profesionales, frente al protagonismo estructural de otros, en lo que se puede considerar un correcto equilibrio de poder durante el proceso (Senabre; Ferran-Ferrer; Perelló, 2018).

La información y comunicación constante que se garantizaron a nivel de todo el centro generó la transparencia, implicación y confianza de todo el personal hospitalario, aseguró su motivación y compromiso, así como su sentido de pertenencia a la organización. Es muy relevante considerar la complejidad de roles que tienen los profesionales sanitarios en cuanto "expertos en sus experiencias" (Sleeswijk-Visser et al., 2005), creadores y no sólo consumidores de contenidos comunicacionales, por lo que su potencial contributivo debe verse potenciado de manera estratégica por la organización (Fischer, 2002).

Por otra parte, contar con un equipo multidisciplinar reforzó el trabajo en equipo, no sólo en el diseño, sino en la implantación y mantenimiento de las redes sociales de la organización sanitaria, garantizando la participación de profesionales activos y no activos en redes durante la campaña del lanzamiento. El liderazgo ejercido por personas expertas y con responsabilidad en la estrategia de comunicación del HURS, así como la presencia del grupo asesor en clave de guía, acompañamiento, apoyo técnico y metodológico (Sanders; Stappers, 2008) aseguró la coordinación y colaboración estructurada durante todo el proceso y fueron clave para la elaboración del modelo.

Las fases identificadas en el mismo, a partir de la experiencia del PSM del HURS, mantienen coincidencias y paralelismos con un modelo estructural descrito por Medina-Aguerrebere (2017) para la gestión de la reputación de marcas hospitalarias, lo que podría confirmar la eficacia del modelo participativo a nivel de diseños estratégicos de comunicación. Además los resultados revelaron la importancia de la profesionalización de la comunicación en el ámbito asistencial, donde es especialmente importante la participación de profesionales expertos en comunicación, así como la implicación de la Unidad de Comunicación para garantizar la calidad y adecuación a las características y necesidades de la organización (Peluchete; Karl; Coustasse, 2016).

Con respecto a la evaluación del impacto PSM, los resultados cuantitativos revelaron una mejora del impacto previsto en todas las redes monitorizadas. Así mismo, el seguimiento a los 8 meses sitúa la presencia del HURS entre los hospitales pioneros en Andalucía, comparando con la situación de otros centros sanitarios públicos y con la tendencia observada en otros estudios (Rando-Cueto; Paniagua-Rojano; De-las-Heras-Pedrosa, 2016).

Entre las limitaciones de este trabajo, es importante referir las complicaciones de gestión de tiempo que surgieron en el desarrollo de las fases metodológicas, así como el condicionamiento de la práctica asistencial, que dificultó la participación en igualdad de condiciones de todo el personal sanitario en las reuniones presenciales. Así mismo, existieron limitaciones propias de la investigación cualitativa en el desarrollo de las entrevistas semiestructuradas.

Sin embargo, los resultados confirman la necesidad de mejorar el uso de las redes sociales como principal acción comunicativa en el marco de la reputación hospitalaria corporativa (Medina-Aguerrebere, 2017), desarrollando planes de comunicación estratégica que planifiquen el uso de medios sociales de manera efectiva, fomentando la interacción e interconectividad de múltiples vías (Tackeray et al., 2012; Heldman; Schindelar; Weaver, 2013) y apostando por la cultura profesional de la comunicación institucional que refuerce el posicionamiento estratégico en el mercado (Medina-Aguerrebere, 2018).

\section{Referencias}

Andersen, Kim-Normann; Medaglia, Rony; Henriksen, Helle-Zinner (2012). "Social media in public health care: Impact domain propositions". Government information quarterly, v. 29, n. 4, pp. 462-469.

https://doi.org/10.1016/j.giq.2012.07.004

Antheunis, Marjolijn L.; Tates, Kiek; Nieboer, Theodoor E. (2013). "Patients' and health professionals' use of social media in health care: Motives, barriers and expectations". Patient education and counseling, v. 92, n. 3, pp. $426-431$.

https://doi.org/10.1016/j.pec.2013.06.020

Bermúdez-Tamayo, Clara; Alba-Ruiz, Rubén; Jiménez-Pernett, Jaime; García-Gutiérrez, José-Francisco; Traver-Salcedo, Vicente; Yubraham-Sánchez, David (2013). "Use of social media by Spanish hospitals: Perceptions, difficulties, and success factors". Telemedicine and e-health, v. 19, n. 2, pp. 137-145.

https://doi.org/10.1089/tmj.2012.0066

Bernhardt, Jay M.; Alber, Julia; Gold, Robert S. (2014). "A social media primer for professionals: Digital dos and don'ts". Health promotion practice, v. 15, n. 2, pp. 168-172.

https://doi.org/10.1177/1524839913517235

Bjerglund-Andersen, Nina; Söderqvist, Thomas (2012). Social media and public health research. University of Copenhagen, Faculty of Science.

http://www.bjerglund.files.wordpress.com/2012/11/final-social-media-and-public-health-research1.pdf

Blázquez-Fernández, Carla; Cantarero-Prieto, David; Pascual-Sáez, Marta (2015). “Promoting the use of health information and communication technologies in Spain: A new approach based on the ICT-H". Icono 14, v. 13, n. 2, pp. $238-259$. https://doi.org/10.7195/ri14.v13i2.860

Calvo-Calvo, Manuel-Ángel (2014). "Calidad y características de los sitios web de los hospitales españoles de gran tamaño". Revista española de documentación científica, v. 37, n. 1. 
https://doi.org/10.3989/redc.2014.1.1049

Castilla, Guillermo (2016). "La comunicación en salud desde el punto de vista de una sociedad médica". Revista española de comunicación en salud, S1, pp. 129-132.

https://doi.org/10.20318/recs.2016.3131

Chetley, Andrew (ed.) (2006). Improving health, connecting people: The role of ICTs in the health sector of developing countries: A framework paper.

http://www.infodev.org/infodev-files/resource/InfodevDocuments_84.pdf

Childs, Lindsey M.; Martin, Christina Y. (2012). "Social media profiles: Striking the right balance". American journal of health-system pharmacy, v. 69, n. 23, pp. 2044-2050.

https://doi.org/10.2146/ajhp120115

Chou, Wen-Ying-Sylvia; Hunt, Yvonne M.; Burke-Beckjord, Ellen; Moser, Richard P.; Hesse, Bradford W. (2009). "Social media use in the United States: implications for health communication". Journal of medical internet research, v. 11, n. 4, e48. https://doi.org/10.2196/jmir.1249

Chretien, Katherine C.; Kind, Terry (2013). "Social media and clinical care: Ethical, professional, and social implications". Circulation, v. 127, n. 13, pp. 1413-1421.

https://doi.org/10.1161/CIRCULATIONAHA.112.128017

Costa-Sánchez, Carmen; Túñez-López, Miguel; Videla-Rodríguez, José-Juan (2016). “Hospitales españoles en la web social. Gestión de Facebook y Twitter por el Hospital Sant Joan de Dèu (Barcelona)". Revista latina de comunicación social, n. 71, pp. 1108-1130.

https://doi.org/10.4185/RLCS-2016-1137

Dizon, Don S.; Graham, David; Thompson, Michael A.; Johnson, Lisa J.; Johnston, Claire; Fisch, Michael J.; Miller, Robert (2012). "Practical guidance: The use of social media in oncology practice". Journal of oncology practice, v. 8, n. 5, pp. $114-24$. https://doi.org/10.1200/JOP.2012.000610

Fischer, Gerhard (2002). "Beyond 'Couch potatoes': From consumers to designers and active contributors". First Monday, v. 7, n. 12.

https://doi.org/10.5210/fm.v7i12.1010

Gomes, Christian; Coustasse, Alberto (2015). "Tweeting and treating: How hospitals use Twitter to improve care". Health care manager, v. 34, n. 3, pp. 203-214. https://doi.org/10.1097/HCM.0000000000000063

Grajales, Francisco J. III; Sheps, Samuel; Ho, Kendall; Novak-Lauscher, Helen; Eysenbach, Gunther (2014). "Social media: A review and tutorial of applications in medicine and health care". Journal of medical internet research, v. 16, n. 2, e13. https://doi.org/10.2196/jmir.2912

Greenhalgh, Trisha; Jackson, Claire; Shaw, Sara; Janamian, Tina (2016). "Achieving research impact through co-creation in community-based health services: Literature review and case study". Millbank quarterly, v. 94, n. 2, pp. $392-429$. https://doi.org/10.1111/1468-0009.12197

Griffis, Heather M.; Kilaru, Austin S.; Werner, Rachel M.; Asch, David A.; Hershey, John C.; Hill, Shawndra; Ha, Yoonhee P.; Sellers, Allison; Mahoney, Kevin; Merchant, Raina M. (2014). "Use of social media across US hospitals: Descriptive analysis of adoption and utilization". Journal of medical internet research, v. 16, n. 11, e264.

https://doi.org/10.2196/jmir.3758

Grindrod, Kelly; Forgione, Andrea; Tsuyuki, Ross T.; Gavura, Scott; Giustini, Dean (2014). “Pharmacy 2.0: A scoping review of social media use in pharmacy". Research in social and administrative pharmacy, v. 10, n. 1, pp. 256-270. https://doi.org/10.1016/j.sapharm.2013.05.004

Heldman, Amy-Burnett; Schindelar, Jessica; Weaver, James B. III (2013). "Social media engagement and public health communication: Implications for public health organizations being truly 'social'". Public health reviews, v. 35, n. 1, e13. https://doi.org/10.1007/BF03391698

Househ, Mowafa (2013). "The use of social media in healthcare: organizational, clinical, and patient perspectives". Studies in health technology and informatics, v. 183, pp. 244-248.

https://doi.org/10.3233/978-1-61499-203-5-244

HURS. Informe anual 2016. Servicio Andaluz de Salud.

https://www.juntadeandalucia.es/servicioandaluzdesalud/hrs3/index.php?id=informes_anuales

Illia, Laura; Balmer, John M. T. (2012). “Corporate communication and corporate marketing: Their nature, histories, differences and similarities". Corporate communications: An international journal, v. 17, n. 4, pp. 415-433.

https://doi.org/10.1108/13563281211274121 
Juárez-Giménez, Juan-Carlos; Fernández-Lisón, Luis-Carlos; Monte-Boquet, Emilio (2014). “Recomendaciones para el uso de las redes sociales para farmacéuticos de hospital (12 consejos que deberías tener en cuenta antes de lanzarte a la Red)". Farmacia hospitalaria, v. 38, n. 2, pp. 86-88.

https://doi.org/10.7399/FH.2014.38.2.7358

Kass-Hout, Taha; Alhinnawi, Hend (2013). "Social media in public health". British medical bulletin, v. 108, n. 1, pp. 5-24. https://doi.org/10.1093/bmb/ldt028

Krowchuk, Heidi V.; Lane, Susan H.; Twadell, Jennifer W. (2010). "Should social media be used to communicate with patients?". MCN The American journal of maternal/child nursing, v. 35, pp. 6-7.

https://doi.org/10.1097/01.NMC.0000366802.88540.54

Lambert, Kristen M.; Barry, Pauline; Stokes Gwen (2012). "Risk management and legal issues with the use of social media in the healthcare setting". Journal of healthcare risk management, v. 31, n. 4, pp. 41-47.

https://doi.org/10.1002/jhrm.20103

Langley, Joe; Wolstenholme, Daniel; Cooke, Jo (2018). “Collective making as knowledge mobilization: the contribution of participatory design in the co-creation of knowledge in healthcare". BMC health services research, v. $18, \mathrm{e} 585$. https://doi.org/10.1186/s12913-018-3397-y

Li, Charlene; Bernoff, Josh (2008). Groundswell: Winning in a world transformed by social technologies. Boston MA: Harvard Business Press. ISBN: 9781422125007

Mairs, Katie; McNeil, Heather; McLeod, Jordache; Prorok, Jeanette C.; Stolee, Paul (2013). "Online strategies to facilitate health-related knowledge transfer: a systematic search and review". Health information and libraries journal, v. 30, pp. $261-277$. https://doi.org/10.1111/hir.12048

Mangold, W. Glynn; Faulds, David J. (2009). "Social media: The new hybrid element of the promotion mix". Business horizon, v. 52, n. 4, pp. 357-365.

https://doi.org/10.1016/j.bushor.2009.03.002

Medina-Aguerrebere, Pablo (2017). “El rol de las redes sociales en la comunicación de marca de los hospitales españoles". adComunica, n. 15, pp. 215-233.

https://doi.org/10.6035/2174-0992.2018.15.11

Medina-Aguerrebere, Pablo (2018). “La gestión de la reputación online de las marcas hospitalarias: una propuesta de modelo". Zer, v. 22, n. 43, pp. 53-68.

https://doi.org/10.1387/zer.17908

Medina-Aguerrebere, Pablo; Buil, Pilar; Heath, Robert L. (2016). “Establishing and demonstrating US hospital brands through Facebook". OBS-Observatorio, v. 10, n. 3, pp 20-40.

https://doi.org/10.15847/obsOBS1032016912

Merchant, Raina M.; Elmer, Stacy; Lurie, Nicole (2011). "Integrating social media into emergency-preparedness efforts". New England journal of medicine, v. 365, n. 4, pp. 289-291.

https://doi.org/10.1056/NEJMp1103591

Moorhead, S. Anne; Hazlett, Diane E.; Harrison, Laura; Carroll, Jennifer K.; Irwin, Anthea; Hoving, Ciska (2013). “A new dimension of health care: Systemic review of the uses, benefits, and limitations of social media for health care professionals". Journal of medical internet research, v. 15, n. 4, e85.

https://doi.org/10.2196/jmir.1933

Peluchette, Joy V.; Karl, Katherine A.; Coustasse, Alberto (2016). “Physicians, patients, and Facebook: Could you? Would you? Should you?". Health marketing quarterly, v. 33, n. 2, pp. 112-126.

https://doi.org/10.1080/07359683.2016.1166811

Rando-Cueto, Dolores (2014). "Presencia y estrategias de comunicación de hospitales andaluces en las redes sociales". Cuadernos artesanos de comunicación, v. 92, pp. 155-173.

http://www.cuadernosartesanos.org/2015/cac92.pdf

Rando-Cueto, Dolores; De-Las-Heras-Pedrosa, Carlos (2016). “Análisis de la comunicación corporativa de los hospitales andaluces vía Twitter". Opción, n. 8, pp. 557-576.

http://www.redalyc.org/articulo.oa?id=31048481033

Rando-Cueto, Dolores; Paniagua-Rojano, Francisco-Javier; De-las-Heras-Pedrosa, Carlos (2016). “Factores influyentes en el éxito de la comunicación hospitalaria vía redes sociales". Revista latina de comunicación social, n. 71, pp. 1170-1186. https://doi.org/10.4185/RLCS-2016-1140

Research Information Network (2010). If you build it, will they come? How researchers perceive and use web 2.0. Research Information Network, London. 
http://www.rin.ac.uk/system/files/attachments/web_2.0_screen.pdf

Richter, Jason; Muhlestein, David B.; Wilks, Chrisanne (2014). "Social media: how hospitals use it, and opportunities for future use". Journal of healthcare management, v. 59, n. 6, pp. 447-461.

https://doi.org/10.1097/00115514-201411000-00011

Rycroft-Malone, Jo; Burton, Christopher R.; Bucknall, Tracey; Graham, lan D.; Hutchinson, Alison M.; Stacey, Dawn (2016). "Collaboration and co-production of knowledge in healthcare: opportunities and challenges". International journal of health policy and management, v. 5, pp. 221-223.

https://doi.org/10.15171/ijhpm.2016.08

Roman, Leah A. (2014). "Using social media to enhance career development opportunities for health promotion professionals". Health promotion practice, v. 15, n. 4, pp. 471-475.

https://doi.org/10.1177/1524839914535213

Sanders, Elizabeth B. N.; Stappers Pieter J. (2008). "Co-creation and the new landscapes of design". CoDesign, v. 4, n. 1, pp. 5-18. https://doi.org/10.1080/15710880701875068

Senabre, Enric; Ferran-Ferrer, Núria; Perelló, Josep (2018). “Diseño participativo de experimentos de ciencia ciudadana". Comunicar, v. 24, n. 54, pp. 23-38.

https://doi.org/10.3916/C54-2018-03

Sleeswijk-Visser, Froukje; Stappers, Pieter J.; Van-der-Lugt, Remko; Sanders, Elizabeth B. N. (2005). “Contextmapping: Experiences from practice". CoDesign, v. 1, n. 2, pp. 119-149.

https://doi.org/10.1080/15710880500135987

Smith, Rachel-Charlotte; Bossen, Claus; Kanstrup, Anne-Marie (2017). "Participatory design in an era of participation". CoDesign, v. 13, n. 2, pp. 65-69.

https://doi.org/10.1080/15710882.2017.1310466

Thackeray, Rosemary; Neiger, Brad L.; Smith, Amanda K.; Van-Wagenen, Sara B. (2012). "Adoption and use of social media among public health departments". BMC public health, v. 12, pp. 242-248.

https://doi.org/10.1186/1471-2458-12-242

Tonia, Thomy (2014). "Social media in public health: Is it used and is it useful?". International journal of public health, v. 59, n. 6, pp. 889-891.

https://doi.org/10.1007/s00038-014-0615-1

Van-de-Belt, Tom H.; Engelen, Lucien J. L. P. G.; Berben, Sivera A. A., Teerenstra, Steven; Samsom, Melvin; Schoonhoven, Lisette (2013). "Internet and social media for health-related information and communication in health care: preferences of the Dutch general population". Journal of medical internet research, v. 15, n. 10, e220.

https://doi.org/10.2196/jmir.2607

Vance, Karl; Howe, William; Dellavalle, Robert P. (2009). "Social internet sites as a source of public health information". Dermatologic clinics, v. 27, n. 2, pp. 133-136.

https://doi.org/10.1016/j.det.2008.11.010

Vanzetta, Marina; Vellone, Ercole; Dal-Molin, Alberto; Rocco, Gennaro; De-Marinis, Maria-Grazia; Rosaria, Álvaro (2014). "Communication with the public in the health-care system: a descriptive study of the use of social media in local health authorities and public hospitals in Italy". Annali dell'Istituto Superiore di Sanità, v. 50, n. 2, pp.163-170.

https://doi.org/10.4415/ANN_14_02_10

Ventola, C. Lee (2014). "Social media and health care professionals: benefits, risks, and best practices". Pharmacy and therapeutics, v. 39, n. 7, pp. 491-499.

https://www.ncbi.n/m.nih.gov/pmc/articles/PMC4103576

Von-Muhlen, Marcio; Ohno-Machado, Lucila (2012). "Reviewing social media use by clinicians". Journal of the American Medical Informatics Association, v. 19, n. 5, pp. 777-781.

https://doi.org/10.1136/amiajnl-2012-000990

Yang, Po-Chin; Lee, Wui-Chiang; Liu, Hao-Yen; Shih, Mei-Ju; Chen, Tzeng-Ji; Chou, Li-Fang; Hwang, Shinn-Jang (2018). "Use of Facebook by hospitals in Taiwan: A nationwide survey". International journal of environmental research and public health, v. 15, n. 6, e1188.

https://doi.org/10.3390/ijerph15061188

Zhang, Wei; Deng, Zhaohua; Evans, Richard; Xiang, Fei; Ye, Qing; Zeng, Runxi (2018). "Social media landscape of the tertiary referral hospitals in China: Observational descriptive study". Journal of medical internet research, v. 20, n. 8, e 249. https://doi.org/10.2196/jmir.9607 


\section{MiBiblioteca}

La revista del mundo bibliotecario

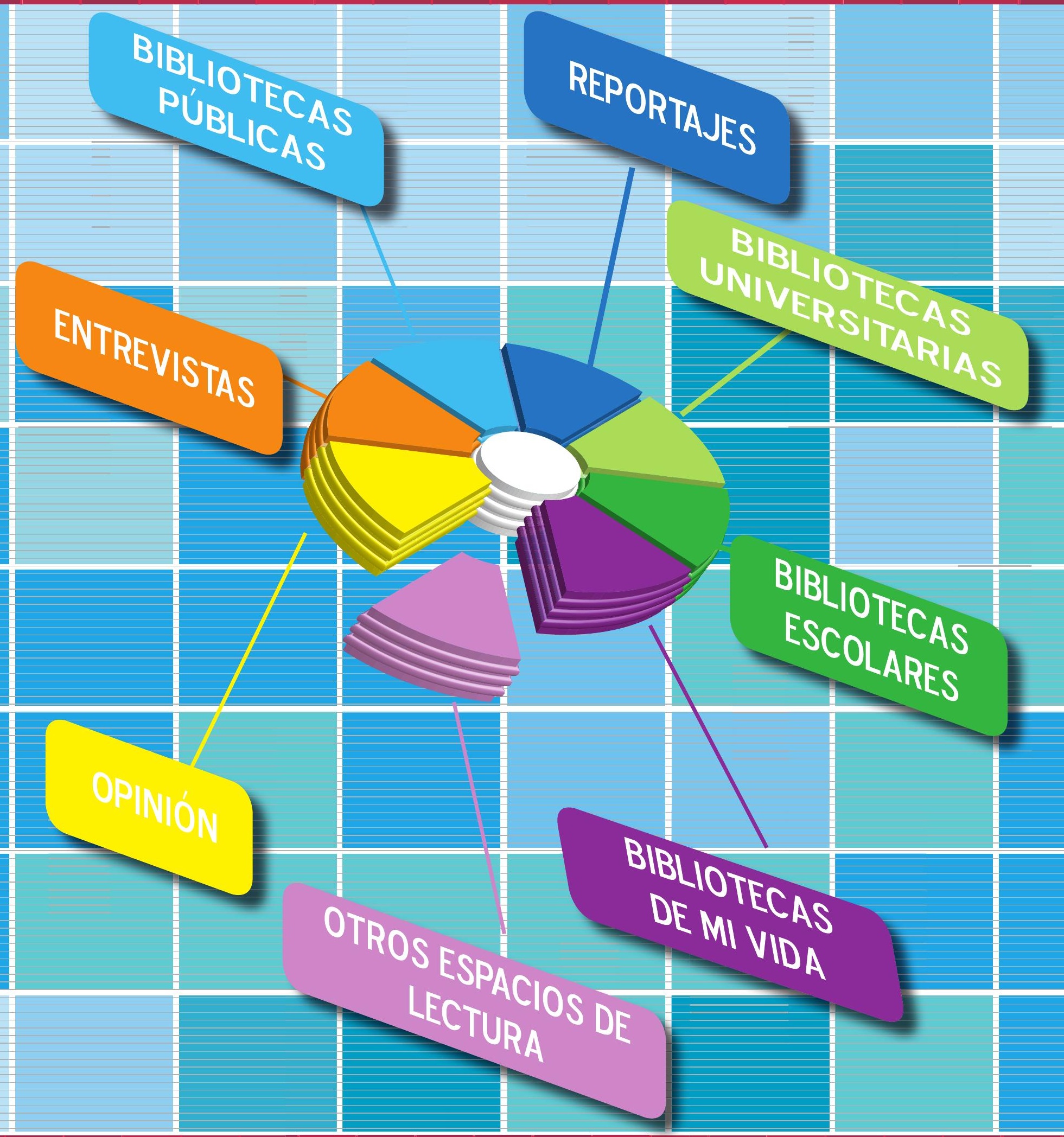

Suscríbete

Por teléfono 952235405 - a través de nuestra web: muw.mibiblioteca.org 